\title{
Transoesophageal cross-sectional echocardiography with a phased array transducer system Technique and initial clinical results
}

\author{
M SCHLUTER, B A LANGENSTEIN, J POLSTER, P KREMER, J SOUQUET^, S ENGEL, \\ P HANRATH
}

From the Department of Cardiology, University Hospital, Hamburg-Eppendorf, West Germany

SUMMARY Cardiac imaging by transthoracic two dimensional echocardiography is impaired in patients with chronic lung disease, those with obesity, and those with abnormal chest wall configuration. In order to overcome these limitations, a miniature phased array ultrasound transducer fitted to the tip of a commercially available gastroscope was developed. Transducer position and orientation can be adjusted completely by external control of vertical displacement inside the oesophagus, rotation, and angulation. Introduction and operation of the transducer gastroscope system are usually well tolerated by the patients, since no mechanical vibrations are generated and there is no need for an oil bag to secure oesophageal wall contact. Cardiac images of high quality are obtained even from structures and regions that are poorly or not at all imaged from external transducer positions.

Two dimensional echocardiography with its wide field of view and high degree of spatial orientation is by now a well established technique in the morphological and functional evaluation of various cardiac disorders. It has frequently been reported, however, ${ }^{1-3}$ that the main limitation of two dimensional echocardiography is the poor image quality obtained in patients with a difficult chest configuration, particularly in those with obesity, emphysema, and chronic obstructive pulmonary disease. Even in normal patients it is sometimes difficult to obtain two dimensional images of diagnostic quality from certain cardiac structures (for example the interatrial septum or the cardiac apex).

The recent development of a miniature ultrasonic phased array probe for transoesophageal imaging of the heart ${ }^{4}$ offers a promising means to circumvent the problems of conventional transthoracic two dimensional echocardiography.

\section{Subjects and methods}

TRANSOESOPHAGEAL TRANSDUCER ARRAY The transducer array for transoesophageal applica-

$\star$ Present address: Advanced Technology Laboratories, Bellevue, Washington, USA.

Accepted for publication 1 April 1982 tions consists of 32 linearly arranged elements with a centre frequency of $3.5 \mathrm{MHz}$, corresponding to a wavelength of $0.43 \mathrm{~mm}$. The total aperture of the array is approximately $9 \mathrm{~mm}$, and a $90^{\circ}$ sector for real time imaging is obtained by appropriate phasing of the single elements. ${ }^{5} 6$ For the contacting surface of the transducer system a spherical acoustic lens was chosen, which gives a depth of field from 2 to $10 \mathrm{~cm}$ in front of the array.

The transducer system is embedded in soft plastic material with carefully rounded edges to avoid damage to the oesophagus, and it is fitted to the distal end of a commercially available gastroscope (Fig. 1). The only rigid part of this new ultrasonic endoscope is represented by the outer dimensions of the transducer array: $35 \mathrm{~mm}$ long, $15 \mathrm{~mm}$ wide, and $16 \mathrm{~mm}$ thick.

Rotation of the gastroscope and transducer angulation is feasible by external control, thus yielding a high degree of freedom for transducer manipulation. In order to operate the transoesophageal phased array scanner, it was connected to a Varian 3400 or $3400 R$ ultrasonic system. All studies were recorded in real time on one-inch videotape.

\section{EXAMINATION TECHNIQUE}

The system was evaluated in 26 patients ( 12 women, 14 men), ranging in age from 22 to 72 years. In order 


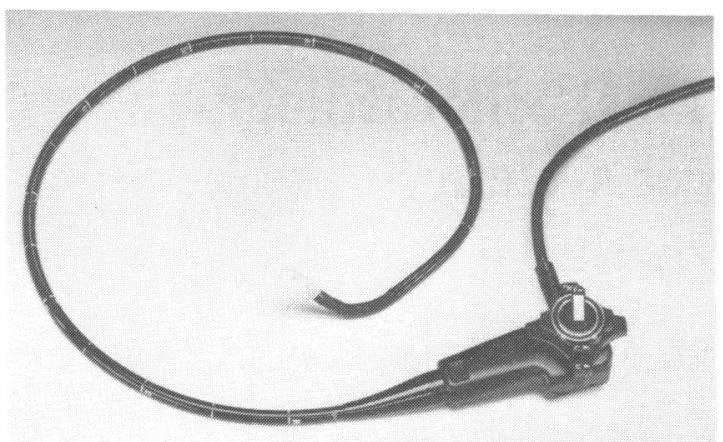

(a)

Fig. 1 (a) The ultrasonic endoscope. A miniature phased array transducer is fixed at the flexible tip of a commercially available gastroscope. Transducer orientation can be adjusted by the external control unit at the proximal end of the gastroscope. (b) Photomontage showing transducer angulation in two orthogonal planes.

to rule out a diverticulum of the oesophagus, all patients had a barium swallow beforehand. Written informed consent was obtained from each patient.

Patients were fasted for about eight hours and were given $0.5 \mathrm{mg}$ atropine sulphate one hour before the investigation in order to avoid bradycardia and hypersalivation. Immediately before the gastroscope was inserted the patients were given $10 \mathrm{mg}$ diazepam intravenously. The transoesophageal study was then performed with the patient supine. Apart from slight gagging no complications were encountered, as the patients usually swallowed the ultrasonic gastroscope as easily as they would a normal endoscopic fibrescope. Cardiac images of high quality were observed when the transducer array was inserted to a level of about $40 \mathrm{~cm}$.

\section{Results}

The first cardiac structure that is readily identified with the gastroscope is the aortic root which is shown in the diastolic frame of Fig. 2. All three cusps are visible, and part of the left ventricular outflow tract as well as the right ventricular outflow tract are clearly outlined. A slight counterclockwise rotation of the gastroscope shows the mitral valve with both leaflets, left atrium and ventricle, and the interventricular septum (Fig. 3). Further descent of the gastroscope allows the left ventricle to be seen in several crosssectional views at different levels of the left ventricu-

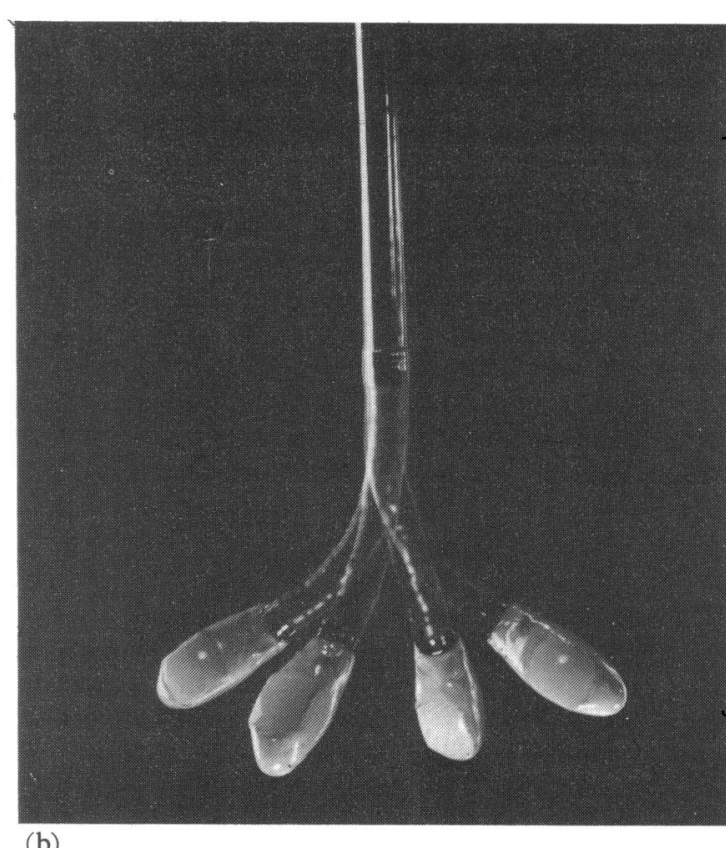

(b)

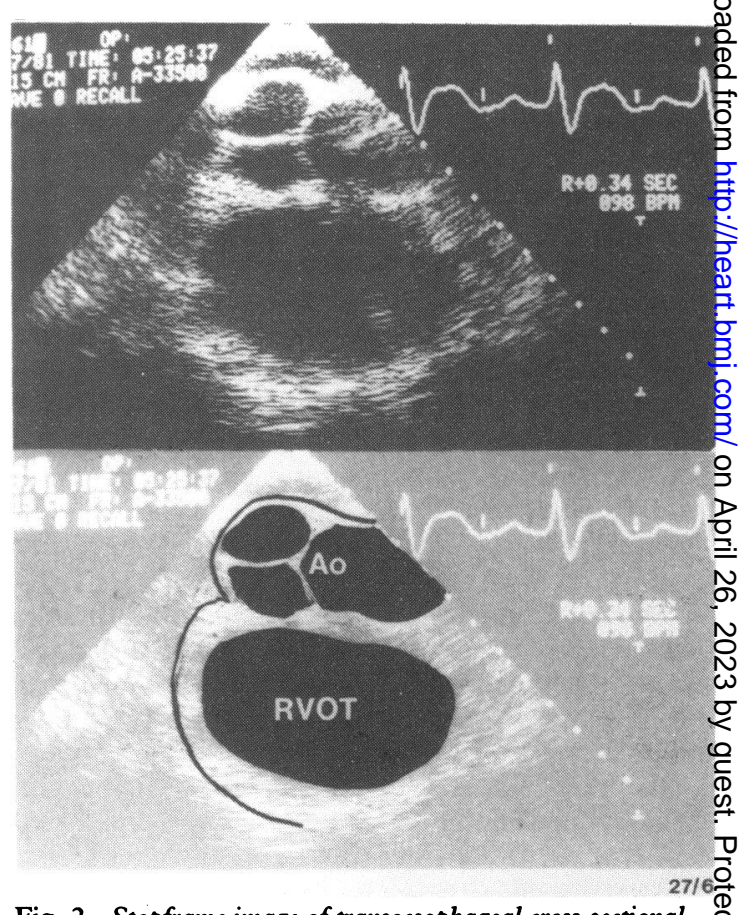

Fig. 2 Stopframe image of transoesophageal cross-sectional echocardiogram at the level of the aortic root during isovolumic $\mathbb{Q}$ contraction. The three cusps are clearly visible, as well as the right ventricular outflow tract (RVOT) and part of the left ventricular outflow tract. 


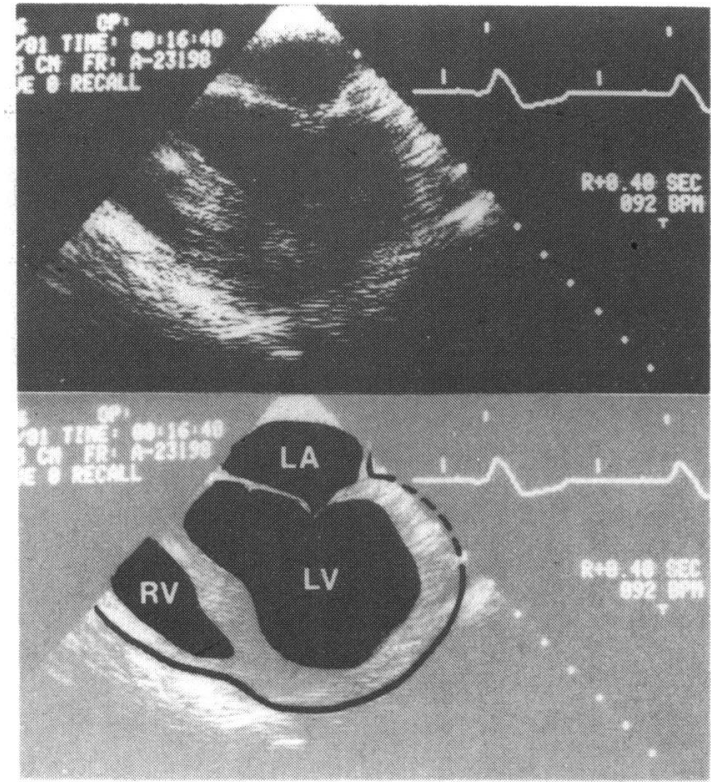

$15 / 23$

Fig. 3 Systolic stopframe image of transoesophageal cross-sectional echocardiogram at the level of the mitral leaflets. Note the proximity of the left atrium $(L A)$ to the ultrasonic transducer at the sector apex, while the left ventricle $(L V)$ and part of the right ventricle $(R V)$ are imaged below the lefi atrivem.

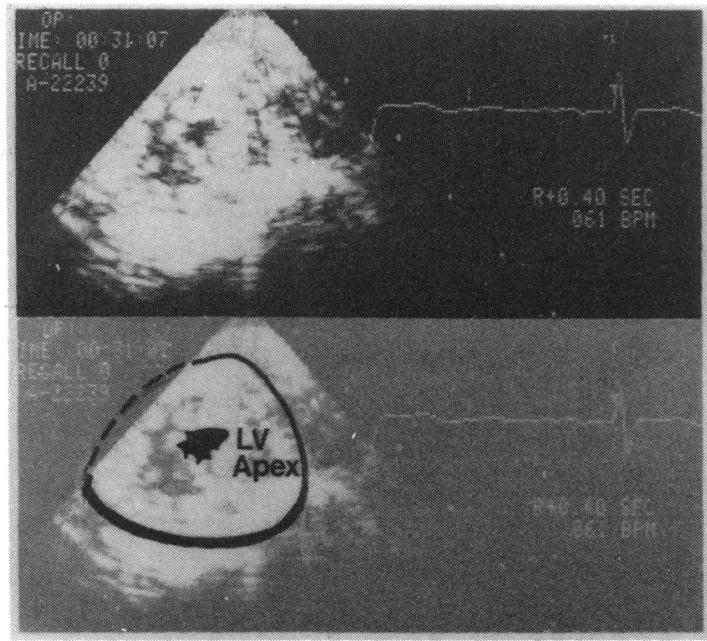

Fig. 5 Transoesophageal short axis cross-sectional view of the left ventricle at the level of the cardiac apex.

lar long axis, for example at the level of the papillary muscles (Fig. 4) or at the cardiac apex (Fig. 5). With a non-dilated left ventricle both ventricles can usually be viewed within the $90^{\circ}$ sector (Fig. 6), thus making it possible to differentiate the right side of the heart by

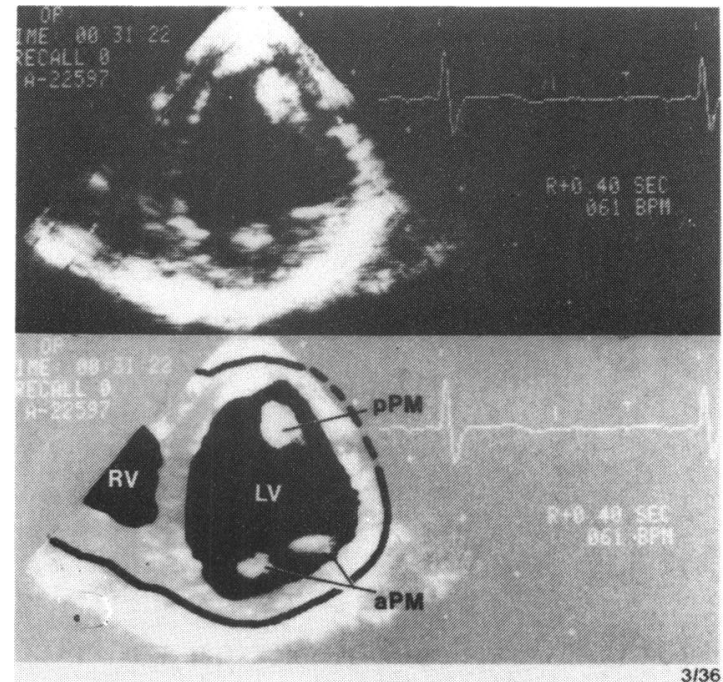

Fig. 4 Transoesophageal short axis cross-sectional view of the left ventricle at the level of the papillary muscles. aPM, anterior papillary muscle; pPM, posterior papillary muscle.

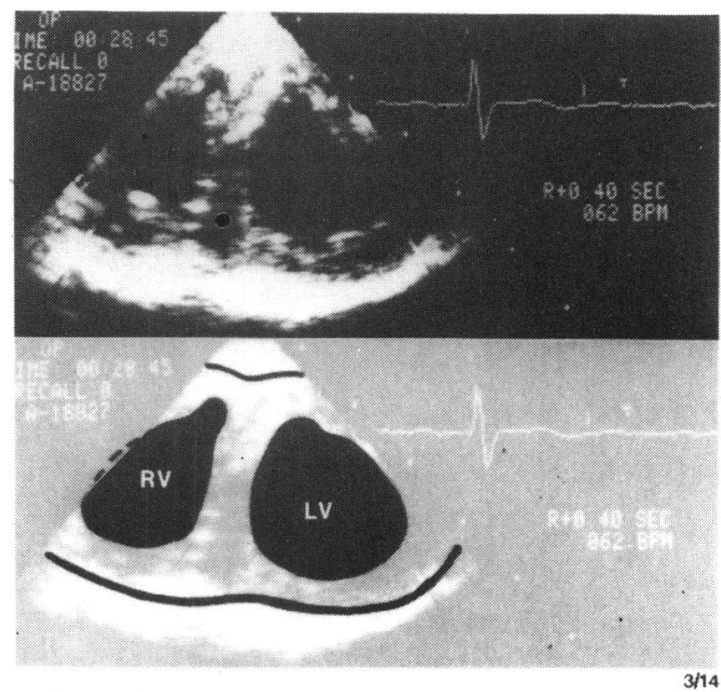

Fig. 6 Transoesophageal cross-sectional view of both ventricles within the $90^{\circ}$ sector scan.

peripheral venous injection of an ultrasonic contrast agent such as agitated saline (Fig. 7).

By clockwise rotation of the gastroscope at the atrioventricular junction the left and right atrium and the tricuspid valve are identified (Fig. 8). In contrast 


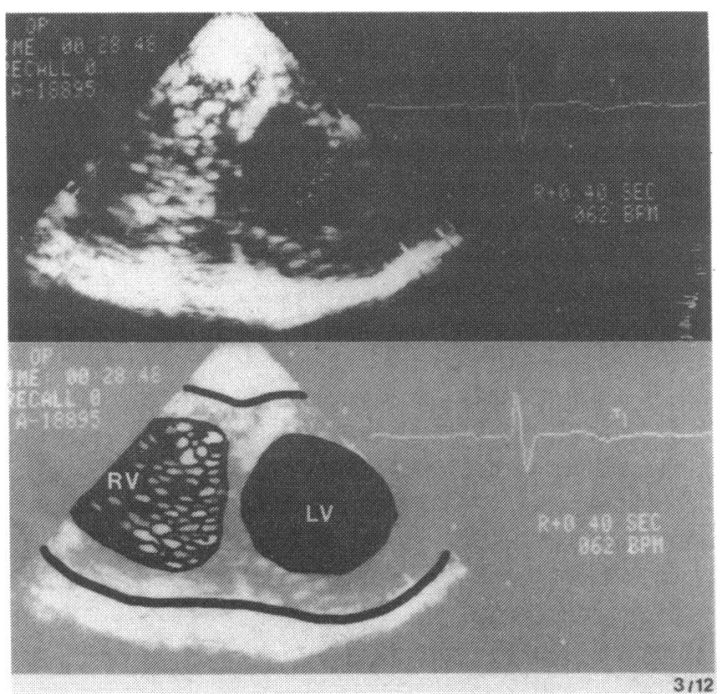

Fig. 7 Same echocardiographic view and same patient as shown in Fig. 6. Because of a peripheral venous injection of agitated saline dense echo contrast appears in the right ventricle.

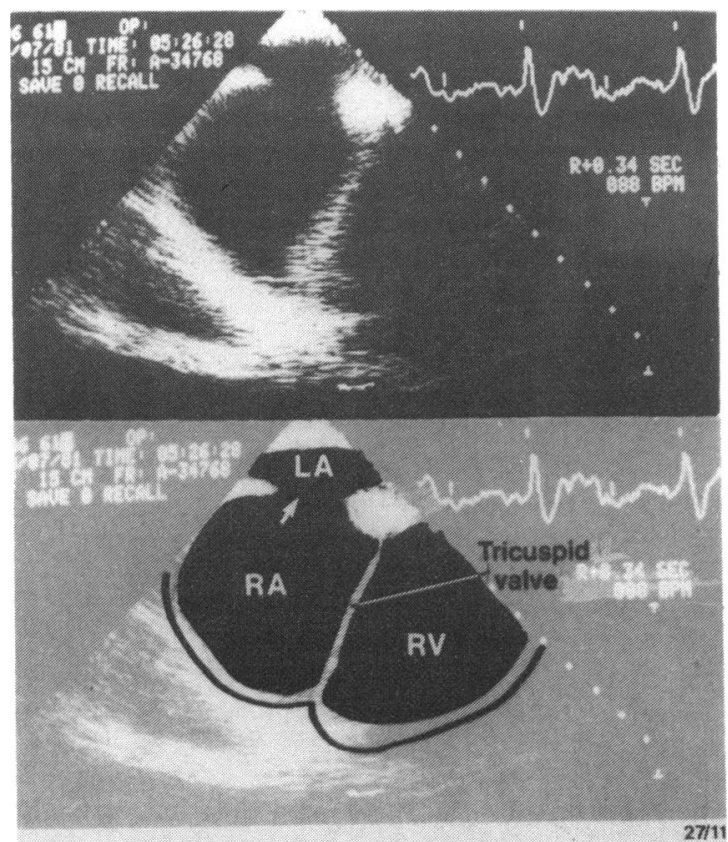

Fig. 9 Transoesophageal cross-sectional view of the right side of the heart in a patient with an ostium secundum type atrial septal defect. The arrow in the lower panel shows the location and the diameter of the defect.

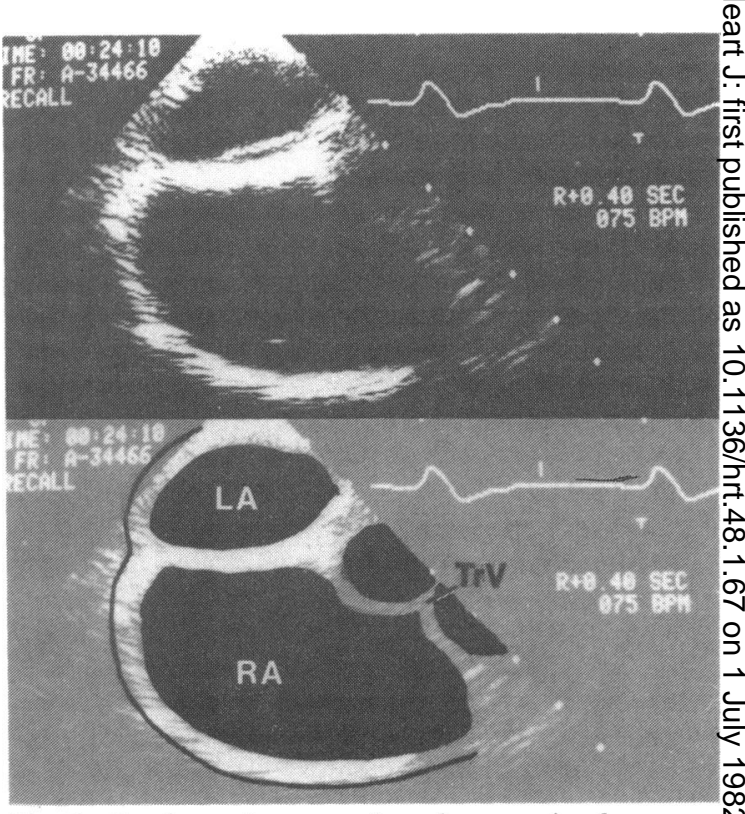

Fig. 8 Stopframe of transoesophageal cross-sectional echocardiogram at the level of the atrioventricular junction. Both atria and the interatrial septum are clearly outlined. The cross-sectional plane dissects the left atrium (LA) cranially making its area appear smaller than that of the right atrium (RA). $\operatorname{Tr} V$, tricuspid valve.

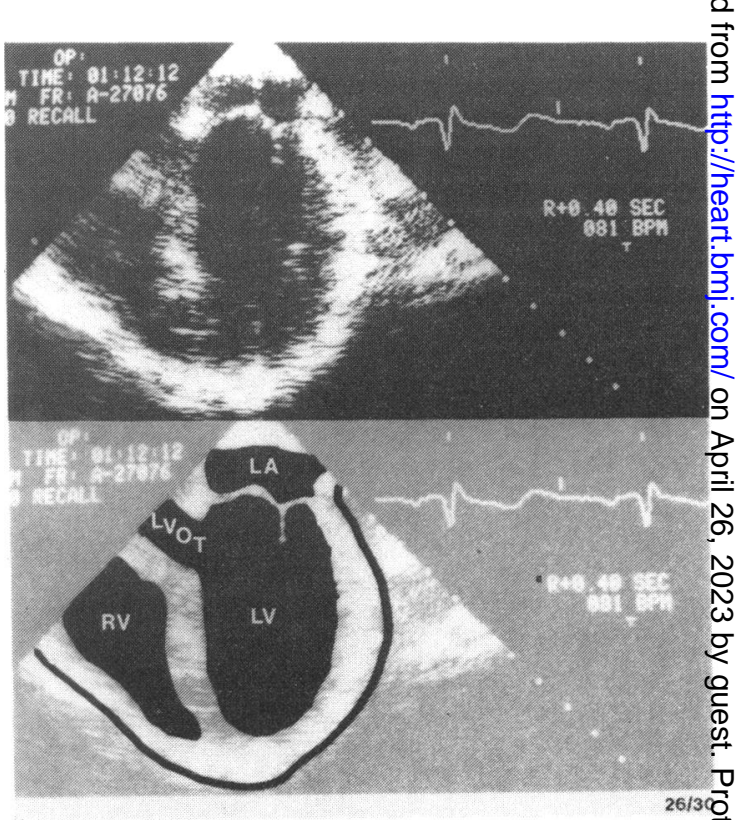

Fig. 10 Two-dimensional transoesophageal echocardiographi $\overrightarrow{\mathbb{D}}$ image from a patient with mitral valve prolapse. The $\frac{\text { के }}{\mathbb{D}}$ cross-sectional plane is shown at the level of the atrioventricula? junction. Note the pronounced late systolic bulging of the anterio mitral leaflet into the left atrium, as compared with the normal closing position of the mitral leaflets in Fig. 3. 
to the transthoracic approach, the interatrial septum is imaged in total without echo drop-outs. This is because the ultrasonic beams hit the interatrial septum nearly perpendicularly from an oesophageal transducer position.

Thus, atrial septal defects can be detected with high diagnostic accuracy, as is the case in the ostium secundum type lesion seen in Fig. 9, where the loss of ultrasonic reflection (arrow in lower panel) corresponds to the location of the defect. Another example of the clinical usefulness of transoesophageal crosssectional echocardiography is the diagnosis of a mitral valve prolapse, exemplified on the systolic frame of Fig. 10. The abnormal arching of the anterior mitral leaflet into the left atrium is clearly documented.

\section{Discussion}

Transoesophageal studies with the aim of assessing cardiac function were first reported in 1971 by Side and Gosling ${ }^{7}$ who performed Doppler velocity measurements in the thoracic aorta. Five years later, Frazin et $\mathrm{al}^{8}{ }^{8}$ presented transoesophageal $\mathrm{M}$-mode recordings with a single element transducer connected to the ultrasound machine by a cable only, which therefore severely restricted ultrasonic beam orientation. Recently, Hisanaga et al. ${ }^{9}$ introduced a high speed rotating ultrasound scanner to obtain cross-sectional cardiac images without hindrance from ribs, sternum, or lung tissue. The transducer housing of this system is enclosed in an inflatable oil bag to secure contact with the oesophageal wall, and it is fixed to the tip of a gastroscope-like shaft that offers no means for controlled manipulation of beam direction. Apart from this disadvantage, the oil bag and the mechanical vibrations of the rotating transducer are liable to cause discomfort to the patient.

These limitations are not present in our system. The $90^{\circ}$ sector is generated by electronic beam steering and there is no need for an oil bag, since oesophageal contact can easily be accomplished by transducer angulation. The attachment of a single element transducer to a normal gastroscope with complete external control of oesophageal transducer orientation has already given promising results in M-mode exercise studies ${ }^{10}$ and pulsed Doppler investigations. ${ }^{11}$ Replacing the single element transducer by a phased array system without a significant increase of gastroscope or transducer dimensions offers new possibilities in cross-sectional cardiac imaging, particularly in cases where transthoracic echocardiography is difficult.

A sector angle of $180^{\circ}$ as in Hisanaga's system cannot be produced by the phased array system, yet the line density within the $90^{\circ}$ sector is obviously higher, and all cardiac structures can be imaged by rotation of the gastroscope and proper angulation of the crosssectional plane. Moreover, introduction and operation of the phased array endoscope cause no discomfort.

In order to obtain satisfactory results with this technique we feel that about two months of gastroscopic practice are necessary, as the success rate very much depends on the skill of the investigator. In the past 15 months, we were able to introduce the gastroscope in 84 out of 103 patients scheduled for one- or two-dimensional transoesophageal echocardiography. The failure rate of $18 \%$ is the result of extremely adverse reactions of some patients to the gastroscopic procedure. In all the other patients, once the transducer gastroscope was swallowed the two dimensional images were always clear. There were no patients with abnormally large left atria compressing the oesophagus, and so the introduction of the gastroscope never caused mechanical irritation of the left atrium.

Transoesophageal cross-sectional echocardiography with a phased array transducer system appears to be of high diagnostic potential in a wide field of clinical applications. Because of the close anatomical relation of the oesophagus and the heart, an improved morphological diagnosis of atrial thrombi, atrial septal defects, mitral valve prolapse, bicuspid aortic valve, or lesions of the tricuspid valve is expected. It will also be possible to detect contraction abnormalities in apical regions of the heart.

Intraoperative monitoring of cardiac function as well as the detection of air-containing microbubbles in the cardiac chambers during myocardial reperfusion after open heart surgery are obvious applications of this new technique, now under study in our laboratory.

Future developments comprise the implementation of two orthogonal arrays or a rotatable array for improved ultrasonic assessment of left ventricular morphology as well as the integration of a fibreoptics system into the ultrasonic endoscope. This will enable visual control of oesophageal transducer position and orientation, thus making the system suitable for abdominal applications as well. This new echocardiographic technique is non-invasive in the sense that blood vessel walls remain intact and no surgery is required, though it is admittedly less pleasant than conventional transthoracic echocardiography. In our experience with more than 80 patients who have undergone transoesophageal one- or two-dimensional echocardiography, there is no risk to the patient.

This work was supported by a grant of the Bundesministerium für Forschung und Technologie.

\section{References \\ 1 Heger JJ, Weyman AE, Wann LS, Dillon JC, Feigen- baum H. Cross-sectional echocardiography in acute}


myocardial infarction: detection and localization of regional left ventricular asynergy. Circulation 1979; 60: 531-8.

2 Visser CA, Lie KI, Kan G, Meltzer R, Durrer D. Detection and quantification of acute isolated myocardial infarction by two dimensional echocardiography. $A m \mathcal{F}$ Cardiol 1981; 47: 1020-5.

3 Stack R, Kisslo J. Evaluation of the left ventricle with two dimensional echocardiography. Am $\mathcal{F}$ Cardiol 1980; 46: $1117-24$.

4 Souquet J, Hanrath P, Zitelli L, Kremer P, Langenstein BA, Schlüter $M$. Transesophageal phased array for imaging the heart. IEEE Trans Biomed Eng, in press.

5 Von Ramm OT, Thurstone FL. Cardiac imaging using a phased array ultrasound system. I. System design. Circulation 1976; 53: 258-62.

6 Kisslo J, von Ramm OT, Thurstone FL. Cardiac imaging using a phased array ultrasound system. II. Clinical technique and application. Circulation 1976; 53: 262-7.

7 Side CD, Gosling RG. Non-surgical assessment of cardiac function. Nature 1971; 232: 335-6.
8 Frazin L, Talano JV, Stephanides L, Loeb HS, Kopel L, Gunnar RM. Esophageal echocardiography. Circula- $C$. tion 1976; 54: 102-8.

9 Hisanaga K, Hisanaga A, Hibi N, Nishimura K, Kambe $\stackrel{\overline{\mathcal{D}}}{\stackrel{\bar{S}}{2}}$ T. High speed rotating scanner for transesophageal cross-sectional echocardiography. Am f Cardiol 1980; 46: 837-42.

10 Kremer P, Hanrath P, Langenstein B, Matsumoto M, Tams C, Bleifeld $W$. The evaluation of left ventricular function at rest and during exercise by transesophageal os echocardiography in aortic insufficiency (abstract). Am $\mathcal{f} \vec{\circ}$ Cardiol 1981; 47: 412.

11 Schlüter $M$, Langenstein BA, Hanrath $P$, Kremer $P, \vec{\omega}$ Bleifeld $W$. Mitral regurgitation detected by transesophageal pulsed Doppler echocardiography (abstract). Eur Heart f 1981; 2, suppl A: 114 .

Requests for reprints to Dr P Hanrath, Department of Cardiology, University Hospital Eppendorf, Mar-으 tinistrasse 52, D-2000 Hamburg 20, West Germany. 